\title{
Star formation onset in baryonic disks: The role of a triaxial halo
}

\author{
P. Mazzei ${ }^{1}$ and A. Curir ${ }^{2}$ \\ 1 Osservatorio Astronomico, Vicolo Osservatorio 5, 35122 Padova, Italy \\ 2 Osservatorio Astronomico, Str. Osservatorio 20, 10025 Pino Torinese, Torino, Italy
}

Received 30 October 2000 / Accepted 12 April 2001

\begin{abstract}
We investigate the effects of the onset of star formation on the growth of bar instability using a smooth particle hydrodynamics code implemented to account for chemo-photometric evolution from UV to nearIR wavelengths. We analyze the role of a non axisymmetric dark matter halo on the bar triggering and the feedback due to the ongoing star formation rate in the disk. We find that the dark matter halo plays a very important role in the evolution of the luminous matter. The star formation rate (SFR) depends indeed both on its mass, which leads the total gravitational field, and on its dynamical state. Stronger initial bursts of star formation are triggered in the more massive unrelaxed haloes than in the relaxed ones, which are also the more concentrated at the beginning. We point out further that the dark matter concentration is different in haloes with a different initial triaxiality ratio, suggesting a dependence of the SFR also on the halo geometry. By mapping the predicted $B$ surface brightness of the new stars formed, we find that a luminous bar along the whole disk develops only in the first stages of such an instability, then later, new stars are born in the inner regions and the bar is reduced to the central 3-4 kpc. After 1.7 Gyr the young stellar component shows stronger bars in the presence of the relaxed haloes with a lower initial triaxiality ratio; strong bars still appear in the old star isodensity contours of the same systems, at variance with our results when star formation is switched off. The formation of new stars causes indeed a lower dynamical coupling between dark matter and baryonic particles, which lengthens the life-time of the bar. Colours and metallicity gradients of new stars allow us to understand deeply the observational consequences of initial geometry and dynamical state of the halo on the star formation process.
\end{abstract}

Key words. galaxies: haloes - evolution - kinematics and dynamics - spiral - stellar content

\section{Introduction}

The bar phenomenon is common among disk galaxies: about one third show a strong bar structure and another one-third have a weaker inner bar. However, their observational properties are strongly dependent on wavelength and spatial resolution achieved (Wozniak et al. 1995; Friedli et al. 1996). Tidal triggering is a possible mechanism of bar formation, since barred galaxies are apparently overabundant in groups and binary systems, although isolated barred galaxies are also known (Martinet \& Friedli 1997). $N$-body simulations show that rotationally supported disks tend to develop instabilities producing long-lived bars, thus it is harder to explain why some disk galaxies do not have bars. However, since this instability can be inhibited if the galaxy has a spherical dark halo and the halo mass inside the disk radius is comparable to or greater than the disk mass (Ostriker \& Peebles 1973; Efstathiou et al. 1982; Christodoulou et al. 1995), much work has been done on the growth of such

Send offprint requests to: P. Mazzei, e-mail: mazzei@pd.astro.it instability inside an analytical (Patsis \& Athanassoula 2000 and references therein) or a non dissipative spherical halo (Sellwood 1981; Athanassoula et al. 1987; Debattista \& Sellwood 2000). The strongly axisymmetric gravitational fields invoked in all these works however are by now seen as unrealistic. This point, which became relevant when the cosmological studies of structure evolution revealed that the shapes of protohaloes are triaxial (Barnes \& Esfhatiou 1987), is by now essential since recent observational constraints point toward triaxial structures of galaxy systems (Sackett 1999). Curir \& Mazzei (1999a; CM99 in the following) have investigated, for the first time in a self-consistent way, how the initial properties of a live halo can influence the development of the bar instability. They carried out SPH simulations embedding a baryonic disk inside rotating haloes of different masses, varying the initial geometry (spherical or triaxial) and dynamical state (relaxed or unrelaxed). However they did not account for the effects of the SFR. Even if such a process implies a large number of uncertainties which can affect the final results, it is nevertheless important to investigate further in order to understand both the bar instability and the 
galaxy evolution. Friedli \& Benz (1995) and Martinet \& Friedli (1997) have studied the effect of star formation on bar instability, although in a very different framework. Their models account for the baryonic component alone, the disk being stabilized by a stellar bulge of different mass ratios as compared to the disk; they focused on the dependence of the results on several parameters, including the rotation of gas with respect to the stars (direct or retrograde), the effect of the cooling processes and their dependence on the initial chemical abundances.

Here we will analyze both how our previous conclusions (CM99) are affected by the onset of star formation and, for the first time, the photometric appearance of the bar allowed by the chemo-photometric implementation of our code already described in Curir \& Mazzei (1999b, Paper II). We find that the ongoing star formation strongly affects stellar morphologies, showing bars also in the presence of more stable conditions previously analyzed. Moreover $B$ and $K$ bars of stars born during the simulations, lasting $1.7 \mathrm{Gyr}$, differ from their isodensity contours which cannot distinguish between very young stars, the main contributors of $U$ and $B$ luminosities, and 1 Gyr old stars, which lead to red and near-infrared luminosities.

We derive a scenario where the DM halo plays a very important role both in triggering the star formation process and on the growth of bar instability. Our chemophotometric approach is a powerful tool to allow further insights into the possible links between dark and luminous matter.

The plan of the paper is the following: in Sect. 2 we summarize the model; simulations performed are described in Sect. 3, where DM and old stars behavior are also analyzed; in Sect. 4 we discuss the gas and the SFR; in Sect. 5 we outline the chemo-photometric properties of the new stars born, i.e. the properties of the bars we have derived using simulated maps, and compare them with the corresponding morphologies; in the following section (Sect. 6) we discuss our main results and in the last section we present our conclusions.

\section{The galaxy model}

The initial configuration is built up as discussed in CM99, here we summarize the main points, the more important parameters and the units used: unit length is $20 \mathrm{kpc}$, mass, $5.97 \times 10^{10} \mathrm{~m}_{\odot}$, time $1.73 \times 10^{8}$ years and velocity $120 \mathrm{~km} \mathrm{~s}^{-1}$.

We use an extended halo (see Sect. 2.1) which is supposed to consist of nondissipative dark matter (DM) particles and a co-rotating exponential disk (see Sect. 2.2) of baryonic matter, gas and stars, with a relative mass ratio 0.1. The value for the gravitational softening (in code units) has been chosen equal to 0.05451. Simulations have been performed with different values of the disk/halo mass ratio, i.e. 0.2 and 1 . We underline that the initial disk configuration takes into account the initial halo potential so that the Toomre $Q$ parameter of the disk takes into account the disk heating due to the halo $(Q=1.5)$.

\subsection{The triaxial halo}

The most probable range of values for the initial triaxiality parameter, $\tau$, of rotating triaxial protohaloes in a Cold Dark Matter Universe (Warren et al. 1992) is 0.6-1 with a peak in the unit value, where:

$\tau=\frac{a^{2}-b^{2}}{a^{2}-c^{2}}, \quad a>b>c$

$a, b$ and $c$ being the axes of the ellipsoid. Using a spin parameter:

$\lambda=\frac{J|E|^{1 / 2}}{G M^{5 / 2}}$

where $E$ is the total energy of the system, $J$ its total angular momentum and $M$ the total mass, Warren et al. (1992) also derived $0.01<\lambda<0.1$ with a peak around the value 0.05 .

In accord with this cosmological framework and with recent observational constraints (Sackett 1999), we adopted DM haloes with initial $\tau$ equal to $0.58,0.84$ and with initial spherical shapes together with $\lambda \simeq 0.07$ to envelope our disk galaxy. Here we carry out a larger set of simulations as compared to CM99 which did not include the $\tau$ value 0.58 , to analyze more throughly the effects of the initial geometry of the halo. We performed simulations using haloes with 20000 or 2000 particles; our results are independent of this choice.

\subsection{The disk}

The baryonic disk is initially described by 4000 or 6000 total particles, 3000 for the gas component and 1000 or 3000 for the nondissipative star component; their relative mass ratio is 0.1 . The total mass of the disk is assumed to be equal to 1 in our units. In the following we will refer to this star component as the old stars to distinguish them from new stars formed from the gas. Simulations using 3000 old star particles show with greater detail features requiring high resolution, such as circumnuclear rings, however, as expected using SPH codes (Navarro \& White 1993; CM99), results vary slightly by increasing this number, in particular as far as the bar is concerned since such a feature extends at least some kpc.

The initial spatial distribution of both gas and stars follows the exponential surface density law.

In Paper II we studied the effects of several important parameters which affect the system evolution when the star formation rate is switched on. We refer the interested reader to that paper for a wide discussion; here we assume a initial mass function (IMF) with a slope, $\alpha=1.5$, extending from 0.1 to $100 \mathrm{~m}_{\odot}$, efficiency $\epsilon=0.4$ (B1 models in Paper II); concerning the effect of SNs on the neighbouring gas particles, only their thermal input has been here included. Following the onset of the star 
Table 1. Properties of the initial configuration of simulations performed.

\begin{tabular}{llllllll}
\hline Simulation $\mathrm{n}$. & Initial state of the halo & $M_{\text {halo }}$ & $t_{\mathrm{dyn}}$ & Initial $\tau$ & $R_{\mathrm{DM}}(r=1)$ & $C$ & $r_{\mathrm{s}}(k p c)$ \\
\hline 1 & RELAXED & 5 & 1.63 & Spherical & 3.15 & 31.2 & 8.50 \\
2 & RELAXED & 5 & 1.73 & 0.58 & 2.19 & 14.1 & 17.9 \\
3 & RELAXED & 5 & 1.88 & 0.84 & 2.02 & 13.5 & 19.4 \\
4 & RELAXED & 1 & 3.65 & Spherical & 0.70 & 20.3 & 7.40 \\
5 & RELAXED & 1 & 3.87 & 0.58 & 0.60 & 13.4 & 11.3 \\
6 & RELAXED & 1 & 4.20 & 0.84 & 0.61 & 9.8 & 15.5 \\
7 & UNRELAXED & 5 & 1.63 & Spherical & 3.91 & 4.6 & 60.0 \\
8 & UNRELAXED & 5 & 1.73 & 0.58 & 3.74 & 2.6 & 60.0 \\
9 & UNRELAXED & 5 & 1.88 & 0.84 & 2.23 & 2.0 & 73.3 \\
10 & UNRELAXED & 1 & 3.65 & Spherical & 0.43 & 2.7 & 60.0 \\
11 & UNRELAXED & 1. & 3.87 & 0.58 & 0.48 & 1.5 & 60.0 \\
12 & UNRELAXED & 1. & 4.20 & 0.84 & 0.35 & 1.2 & 73.3 \\
\hline
\end{tabular}

Where $M_{\text {halo }}$ and $t_{\text {dyn }}$ are in our units (Sect. 2).

Table 2. System properties at $t=9$.

\begin{tabular}{lllllll}
\hline Simulation $\mathrm{n}$. & Initial state of the halo & $M_{\text {halo }}$ & Initial $\tau$ & $(b / a)_{\text {old }}$ & $C$ & $r_{\mathrm{s}}(\mathrm{kpc})$ \\
\hline 1 & RELAXED & 5 & Spherical & 0.37 & 36.3 & 7.3 \\
2 & RELAXED & 5 & 0.58 & 0.44 & 29.2 & 8.7 \\
3 & RELAXED & 5 & 0.84 & 0.52 & 27.4 & 9.6 \\
4 & RELAXED & 1 & Spherical & 0.42 & 37.2 & 4.1 \\
5 & RELAXED & 1 & 0.58 & 0.50 & 25.0 & 6.1 \\
6 & RELAXED & 1 & 0.84 & 0.50 & 29.7 & 5.2 \\
7 & UNRELAXED & 5 & Spherical & 0.42 & 32.0 & 8.7 \\
8 & UNRELAXED & 5 & 0.58 & 0.30 & 28.5 & 9.7 \\
9 & UNRELAXED & 5 & 0.84 & 1. & 24.8 & 11.2 \\
10 & UNRELAXED & 1 & Spherical & 0.52 & 12.9 & 12.6 \\
11 & UNRELAXED & 1. & 0.58 & 0.45 & 11.9 & 13.7 \\
12 & UNRELAXED & 1. & 0.84 & 0.5 & 11.1 & 14.8 \\
\hline
\end{tabular}

formation the final number (i.e. $t=10$ ) of baryonic particles is strongly increased ranging between a minimum of 7010 up to 21300 particles.

\section{Formation of the bar structure}

In order to give insights into the role of the dynamical state of the halo on the baryonic disk, we merge these two components as presented in CM99:

- in the unrelaxed cases, we merge the two components together following both the consequences of the gravitational instability of the halo on the baryonic disk and the reaction of the DM component to the baryonic matter;

- in the relaxed cases, we merge the disk inside the relaxed halo, i.e. the halo configuration after 5 dynamical times, where we define the dynamical time as: $t_{\mathrm{dyn}}=\sqrt{\frac{3 \pi}{16 G \rho}}$.
The initial $T / W$ ratio is always lower than the Ostriker \& Peebles threshold value for the onset of bar instability.

By comparing the results of both these methods, we disentangle the reaction of the halo due to the disk immersion from that due to its gravitational collapse. At the same time we analyze the responsive disk in these different situations with particular attention to outline the more suitable conditions for triggering bar instabilities.

\subsection{The $D M$}

Since we have haloes with both different masses and different initial axis lengths, we have different dynamical timescales (see Table 1).

Following Navarro et al. (1995) we define the halo concentration, $C$ (Table 1 ), as the ratio between $r_{200}$, i.e. the radius where the DM density overcomes the critical density (given by $3 H_{0}^{2} / 8 \pi G$ with $H_{0}=50 \mathrm{~km} \mathrm{~s}^{-1} / \mathrm{Mpc}$ ) by a factor of 200 , and $r_{\mathrm{s}}$, i.e. the inner radius where the halo density departs from a isothermal density distribution 
(i.e. $\left.\rho \propto r^{-2}\right)$. At the initial time $r_{\mathrm{s}}$ is just the average radius of the system (Sect. 2.1 ).

By comparing the values of $C$ at the beginning and at the end of evolution (Tables 1 and 2 respectively) we find that the halo concentration depends on its initial triaxiality ratio, so that the more concentrated the halo, the lower this ratio. We notice that our systems, which become more concentrated with time as a consequence of the gravitational instability induced by the embedded disk, show in Table 2 the same trend as in Table 1. However, their final concentration depends also on the halo mass itself which settles the dynamical time scale of the system. So the less massive unrelaxed haloes, which show lower initial concentrations (Table 1), have also lower final concentrations.

We point out further that after $1.7 \mathrm{Gyr}$, density profiles in the inner regions $\left(\rho \propto r^{-\gamma}, r \leq r_{\mathrm{s}}\right)$ of unrelaxed haloes are steeper $(\gamma \simeq 1.6$, i.e. power law $)$ and those of relaxed ones are shallower $(0.3 \leq \gamma \leq 1$, i.e. core-like) than expected for "normal" relaxed systems $(\gamma \simeq 1$; Navarro et al. 1995; see also Paper II). Thus we derive that the effect of the instability induced by the baryonic disk is to modify the halo density distribution in the inner regions by producing a DM core-like density profile, as consequence of a further process of relaxation, or a DM power law profile, as consequence of a stronger one.

The ratio of DM to baryonic mass inside the disk's radius at $t=2, R_{\mathrm{DM}}(r=1)$, is reported in Table 1 ; a near constant value of $R_{\mathrm{DM}}(r=1)$ is achieved after $t=2$, as in CM99. Results by Athanassoula et al. (1987) suggest a value of $R_{\mathrm{DM}}(r=1)$ larger than 0.81 to inhibit the $m=1$ (i.e. lopsided) instability and larger than 2.2 to suppress the $m=2$ (i.e. two arms) instability. However, these critical values were deduced using the results of Toomre (1981) which neglect any deviation from axisymmetry. We will discuss the meaning of such values, which reflect on stellar morphology, in the next section.

\subsection{The old stellar component}

Stellar disks embedded both in the less massive haloes and in the more massive, unrelaxed ones cannot re-arrange quickly and acquire a bar structure, lasting until the end of the simulation, even if their $R_{\mathrm{DM}}(r=1)$ values are beyond the instability threshold suggested by Athanassoula et al. (1987); we find that this result is independent of the star formation onset.

However, relaxed massive haloes have a stabilizing action on the non dissipative disk particles which re-arrange in a nucleus + disk configuration before the end of the simulation (i.e. 1.7 Gyr) if the SFR is switched off. Thus, in that case the more massive systems drive a different reaction of the baryonic disk depending on the initial dynamical state of their halo. Figure 1 (top panels) shows that the boxy isodensity contours, which characterize the final $x-y$ morphology in such a system (right panel), translate into a bar structure following the effect of the star formation onset (left panel); Fig. 2 shows the time evolution of isodensity contours of the same simulation. Thus if the SFR is switched on, old stellar bars live until the end $(t=10)$ in all the simulations performed here.

Friedli \& Benz (1995) also found a similar result by analyzing the effects of the SFR, however in a very different framework as we will discuss further in Sect. 6 .

Martin (1995) and several other studies such as those by Wozniak et al. (1995); Friedli et al. (1996); Martin \& Friedli (1997, 1999) and Martinet \& Friedli (1997), use the axis ratio $(b / a)$ as a quantitative parametrization of the bar strength; this is a very useful definition to compare models with observations for a given resolution and density contrast achieved. We find that after about $1.6 \mathrm{Gyr}$ the bar strength (Table 2) correlates with the mean density of the whole system inside the disk radius, which can be derived from the value of $R_{\mathrm{DM}}(r=1)$ in Table 1: stronger bars (i.e. $b / a<0.6$; Martinet \& Friedli 1997) develop in stellar disks embedded in the more concentrated haloes which have also the lower initial triaxiality ratio, $\tau$. Unrelaxed systems require longer times to attain the same dynamical stage, thus, it is not surprising that they deviate slightly from the previous trend. We point out that values of $b / a$ in Table 2 have been computed with the same resolution $(600 \mathrm{pc})$ and density contrast (8) for all the simulations; different choices can change the values but not the trend outlined.

\section{The star formation rate}

It is well known that the density of the gas is the main factor determining the SFR, but we find that there are several other unexpected quantities which also can affect it, in particular the initial dynamical state of the halo, its total mass and its initial geometry. Our simulations show that the initial burst of SFR is enhanced inside unrelaxed haloes (Fig. 3). Thus the effect of the mass of the halo, i.e. of the gravitational field, is modulated by the dynamical conditions of the halo itself. Furthermore, more massive relaxed systems achieve the lower SFRs while the more massive unrelaxed states lead to higher SFRs (Table 3, Cols. 2 and 7). For a given mass of the halo, relaxed systems hold, from the beginning, denser DM cores than the unrelaxed ones. As we will discuss below, such high DM density leads to less favorable star forming conditions. DM haloes react to the disk immersion by collapsing again, thus raising their density further. Table 1 shows that both the initial concentration, $C$ (see Sect. 3.1), and $R_{\mathrm{DM}}(r=1)$, i.e. the average $\mathrm{DM}$ density inside the disk radius, are at least double in relaxed haloes than in the unrelaxed ones. Moreover, for relaxed states, both these quantities which measure the depth of the potential well, increase by reducing the initial triaxiality of the halo. Thus both the strength of the initial burst of star formation and the global activity of the star formation are also affected by the initial geometry of the halo.

Using the $Q_{\mathrm{g}}$ definition given in Friedli \& Benz (1993), we show that the more concentrated DM systems heat the gas in the disk through viscous effects from the beginning 


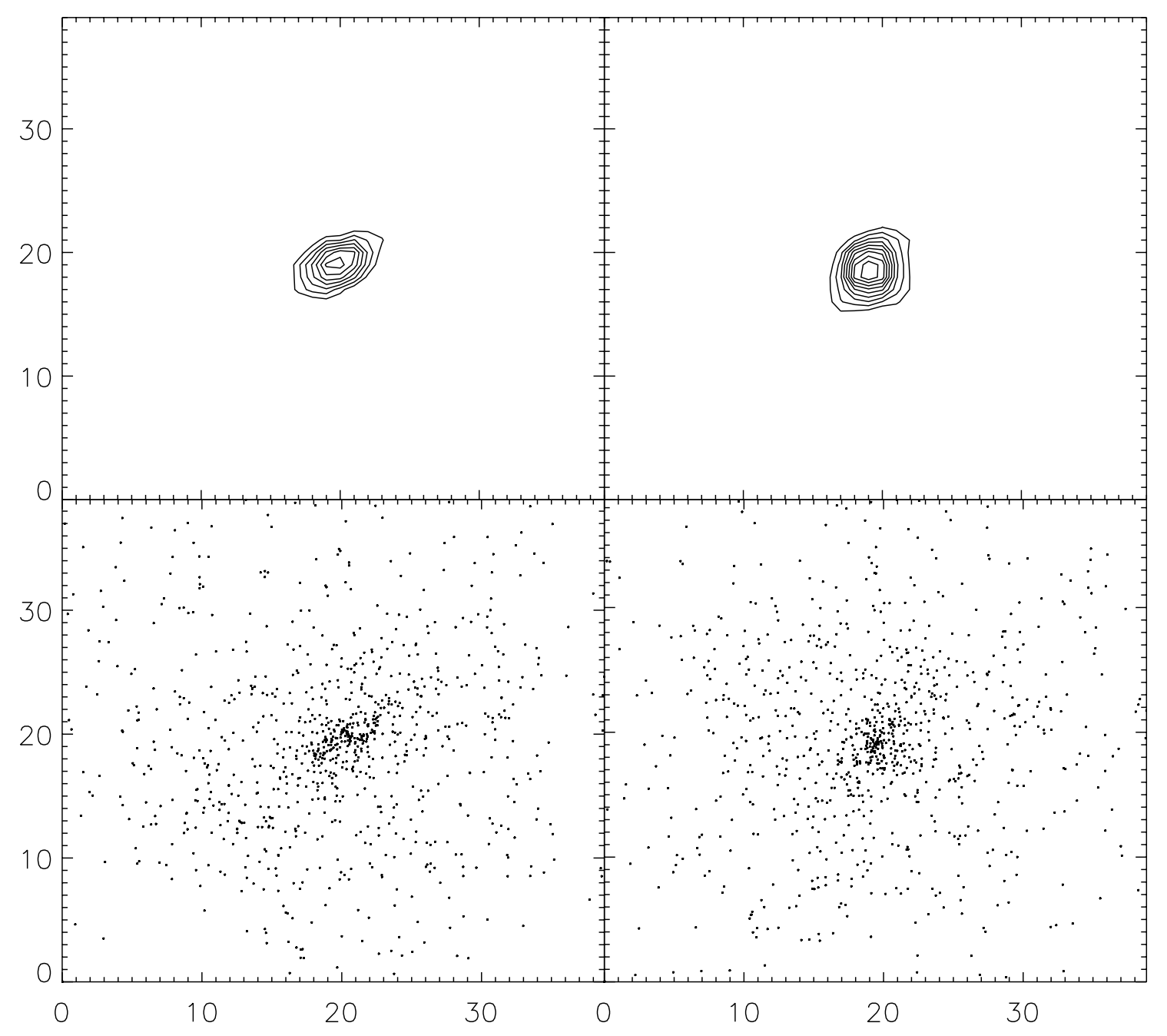

Fig. 1. Upper (lower) panels show 15 isodensity contours (morphologies) at $t=10, x-y$ projection, for the old star component of simulation 1 (Table 1): left column with the star formation switched on, right column with the star formation switched off; the spatial range is $30 \times 30 \mathrm{kpc}$ and the spatial resolution is $750 \mathrm{pc}$; the density contrast achieved, 4.2, corresponds to a lower density contour 3 times higher than the minimum density contour allowed by the map.

more than other systems. Figure 4 compares the radial distribution of $Q_{\mathrm{g}}$ at different times for simulations 2 and 8 which have the same initial geometry but different dynamical states of a massive halo. The more concentrated the global system, the hotter the disk, i.e. $Q_{\mathrm{g}}>1$, due to the effects both of viscous friction, enhanced with the DM concentration, and of SN explosions following the onset of star formation, which reduces the density of the gas by heating neighbouring clouds. Thus conditions for star formation, i.e. $Q_{\mathrm{g}}<1$ (Friedli \& Benz 1993), which initially are less favourable in the more concentrated systems (Fig. 4), become even less favourable during their evolution: star formation is confined to their inner regions, and slows down quickly. Table 3 (Col. 2) shows that on average the initial burst of star formation is higher in the unrelaxed massive systems than in the relaxed ones. This lasts $0.2-0.1 \mathrm{Gyr}$, then the SFR slows down and after 1.7 Gyr ranges from $2.5 m_{\odot} /$ yr to $0.1 m_{\odot} /$ yr. Higher SFRs may occur in the later times of more concentrated haloes, given their larger residual gas fraction.
The gas morphology does not differ strongly from that described by CM99 even if the inner regions (i.e. $r<5 \mathrm{kpc}$ ) are now strongly depleted of gas. However we have further improved the grid of models here compared to those by CM99: for a given mass of the halo, $M$, we are comparing systems with almost the same dynamical time (they differ from each other by less than 8\%) as $\tau$ is increasing. Thus the dependence on the initial geometry of the halo is now more firmly settled.

Relaxed cases with $M=1$ show a gaseous bar which disappears within $t=7 \simeq 8$, then the system re-arrange in a disk around a small nucleus (SBc) which holds almost $20 \%-30 \%$ of the total mass of gas; Table 3 (Col. 7) shows that at the final time such a mass is reduced by $50 \%-$ $70 \%$ compared with the corresponding cases without star formation; however, the amount of gas in the inner regions is less than $70 \%-90 \%$ compared with the same cases.

When $M=5$ the gaseous bar lasts until $t=5 \simeq 5.5$, then degenerates in a disk with better defined spiral arms if the initial triaxiality ratio of the system is higher 

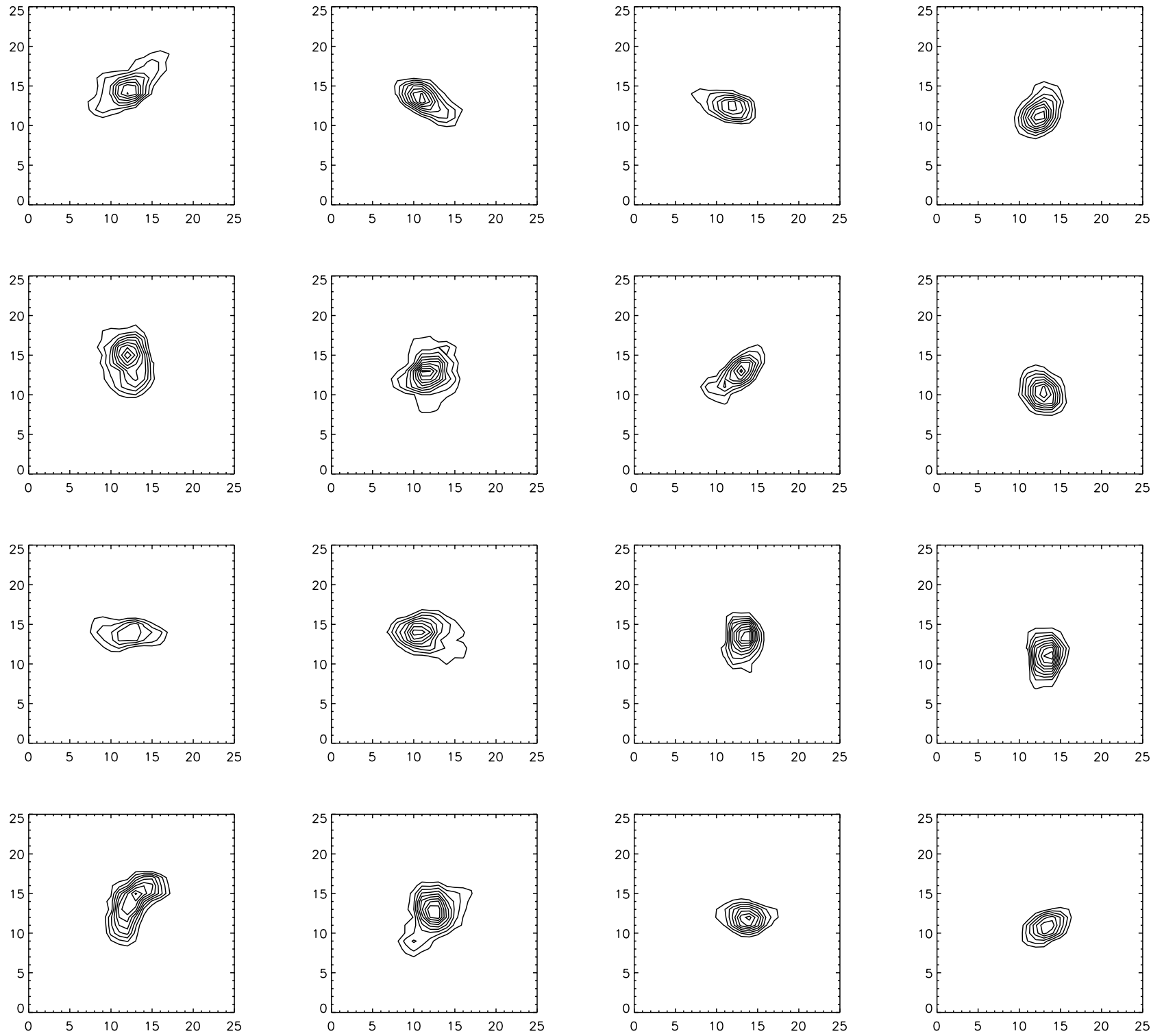

Fig. 2. Time evolution of isodensity contours, 15 levels ( $x-y$ projection), for the old stars of simulation 1 in Table 1 ; time increases from top to bottom and left to right with step of 0.5 from $t=1.5$ to $t=7.5$ in our units, the last 3 panels in the right column correspond to $t=8,9$ and 10 respectively; the density contrast achieved, 4 ., corresponds to a lower density contour at least 3 times higher than the minimum density contour allowed by the map at each time; the spatial range is $20 \times 20 \mathrm{kpc}$ and the spatial resolution $770 \mathrm{pc}$.

(Figs. 5 and 6); at the same time, these hold a dense nucleus with about $40 \%$ of the total mass of gas. The gas depletion in the inner regions ranges from $14 \%-20 \%$ for simulation 2 and 1 (Figs. 6 and 5 respectively), to $69 \%$ for simulation 3 .

Unrelaxed cases with $M=1$ show a gaseous bar around a very small nucleus lasting until the end of simulations; these include $35 \%-47 \%$ of the residual mass of gas. In the inner regions the gas is reduced on average by $85 \%$ compared with the same cases without star formation. When $M=5$ the barred configuration, which lasts until the end of simulations, is more clearly defined in the initial spherical model and is made fainter by increasing the initial triaxiality ratio; this holds from $42 \%$ to $46 \%$ of the available mass of gas which is reduced by $53 \%-66 \%$ compared with the cases where the star formation is switched off.

\section{Chemo-photometric predictions}

Figures 7 and 8 compare morphologies of new stars in simulations 2 and 8: after 1.7 Gyr new stars are still arranged along a central bar structure which appears more clearly defined in relaxed than in unrelaxed systems. With the aim of analyzing more deeply bar properties of new stars in our models, we have compared $B$ and $K$ maps of all the simulations at the final time using the same spatial resolution and magnitude contrast (see Figs. 9 and 10 for an example). We find that bars are more easily detected at short wavelengths; moreover in the $B$ band, the 

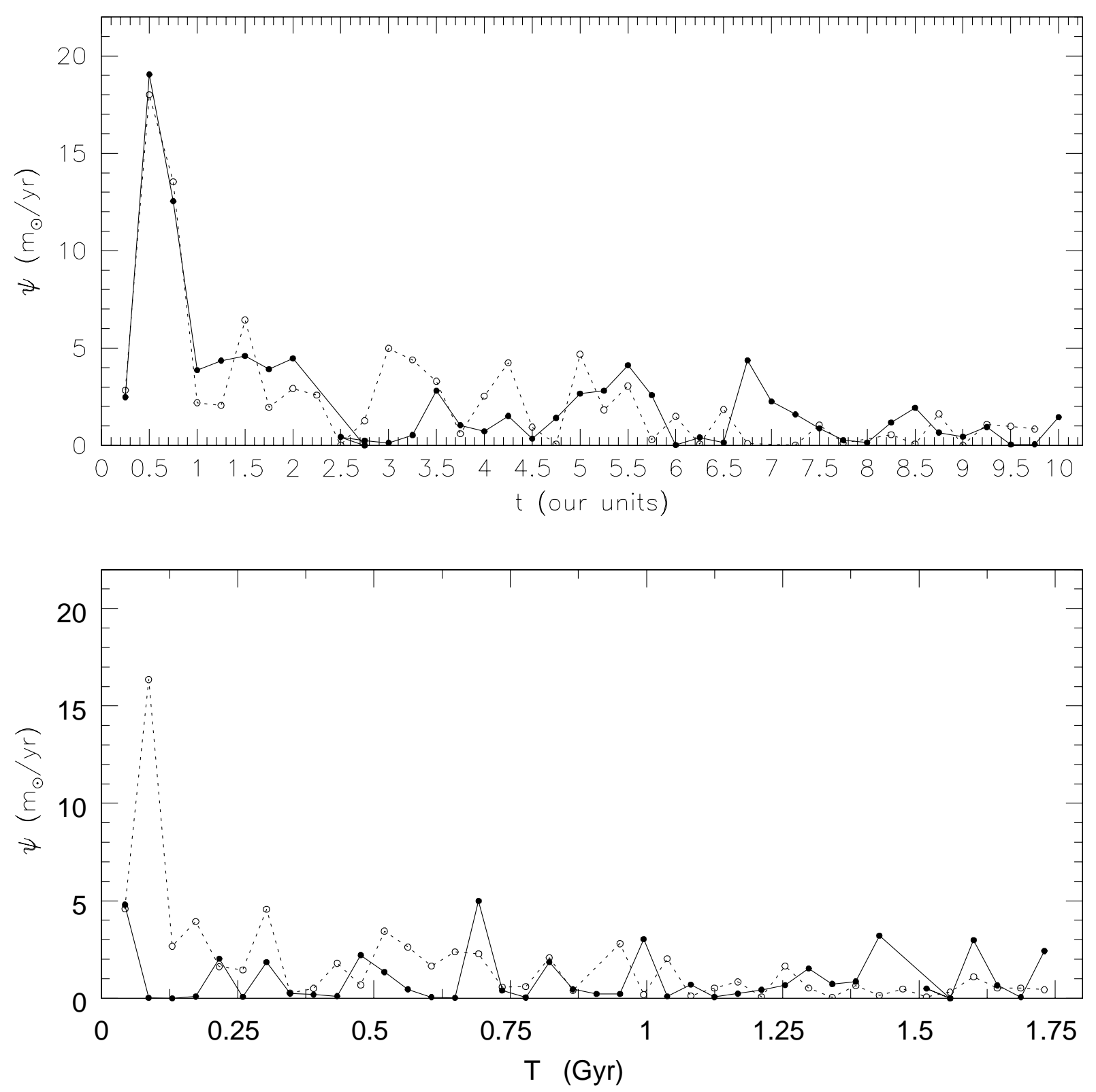

Fig. 3. Top panel shows the SFR at different time steps for simulation 7, continuous line, and 8, dotted line, in Table 1; bottom panel for simulation 1 , continuous line, and 2, dotted line, at the corresponding times in Gyr.

strength of the bar correlates with the system concentration, $C$, i.e. stronger bars prefer more concentrated systems (Table 3); at the same wavelength bars in bars are more common in the less massive unrelaxed systems. We point out that different SFRs can strongly modify the $B$ band appearance of a system for a given spatial resolution and magnitude contrast. Therefore a burst of star formation can reduce a galaxy's dimension down to $3 \mathrm{kpc}$ both in the early phases of evolution in more concentrated systems and in the later stages of the other systems. This affects the $U$ band appearance even more strongly. In the $K$ band (Fig. 10 lower panels) the system appears larger than at short wavelengths ( $U$ and $B$ bands). This is because the infrared light is less dependent on the actual value of the SFR, as shown in Fig. 11, where $B$ luminosities and $B-K$ colors change more than $K$ luminosities and $J-K$ infrared colors. Figure 12 shows the corresponding spectral energy distributions (SEDs, continuous lines) at $t=9$. We have assumed that the SED of the old stellar component is the same as a normal disk galaxy i.e. as derived by the chemo-photometric evolutionary population synthesis models (Mazzei et al. 1992) which satisfactorily fit the SED of normal galaxies, such as our own galaxy. The total SED of our simulated system is composed of the SEDs of its two stellar components which we link together, assuming that the SFR of the old stellar disk is the same as in our own galaxy (i.e. $\simeq 3 m_{\odot} / \mathrm{yr}$, Mazzei et al. 1992). It is indeed well known that the UV luminosity (i.e. $\lambda=0.13 \mu \mathrm{m})$ is proportional to the SFR. Since the total mass of new stars is at least 15 times lower than that of old stars, it is not surprising that $K$ luminosity is almost completely due to the old stellar component. 
Table 3. Properties of the new star component after 1.6 Gyr (i.e. $t=9$ ).

\begin{tabular}{ccccccc}
\hline $\mathrm{N}$ & $S F R_{\max }\left(m_{\odot} / \mathrm{yr}\right)$ & $b / a$ & $B-K$ & $L_{B}\left(10^{10} L_{\odot} / \mu \mathrm{m}\right)$ & $R_{\text {opt }}(\mathrm{kpc})$ & $M_{\text {stars }} / M_{\text {gas }(t=0)}$ \\
\hline 1 & 4.8 & 0.50 & 1.17 & 0.305 & 0.52 & 0.26 \\
2 & 16.4 & 0.60 & 2.38 & 0.168 & 3.27 & 0.46 \\
3 & 19.1 & 0.71 & 2.45 & 0.147 & 1.70 & 0.44 \\
4 & 10.3 & 0.40 & 2.33 & 0.225 & 1.20 & 0.55 \\
5 & 16.4 & 0.60 & 2.60 & 0.155 & 2.39 & 0.53 \\
6 & 8.0 & 0.63 & 2.39 & 0.158 & 1.66 & 0.44 \\
7 & 19.1 & 0.50 & 2.12 & 0.331 & .505 & 0.64 \\
8 & 18.0 & 0.70 & 2.39 & 0.240 & 1.19 & 0.68 \\
9 & 19.4 & 0.80 & 1.98 & 0.277 & .870 & 0.51 \\
10 & 14.6 & 0.60 & 2.79 & 0.156 & 4.84 & 0.59 \\
11 & 15.4 & 0.60 & 2.56 & 0.199 & 3.52 & 0.61 \\
12 & 14.0 & 0.71 & 2.96 & 0.131 & 3.75 & 0.59 \\
\hline
\end{tabular}

$R_{\text {opt }}$ marks the radius containing $80 \%$ of the new star $B$ luminosity;

$b / a$ is derived from $B$ maps using the same magnitude contrast, spatial resolution and spatial range as described in Fig. 9.
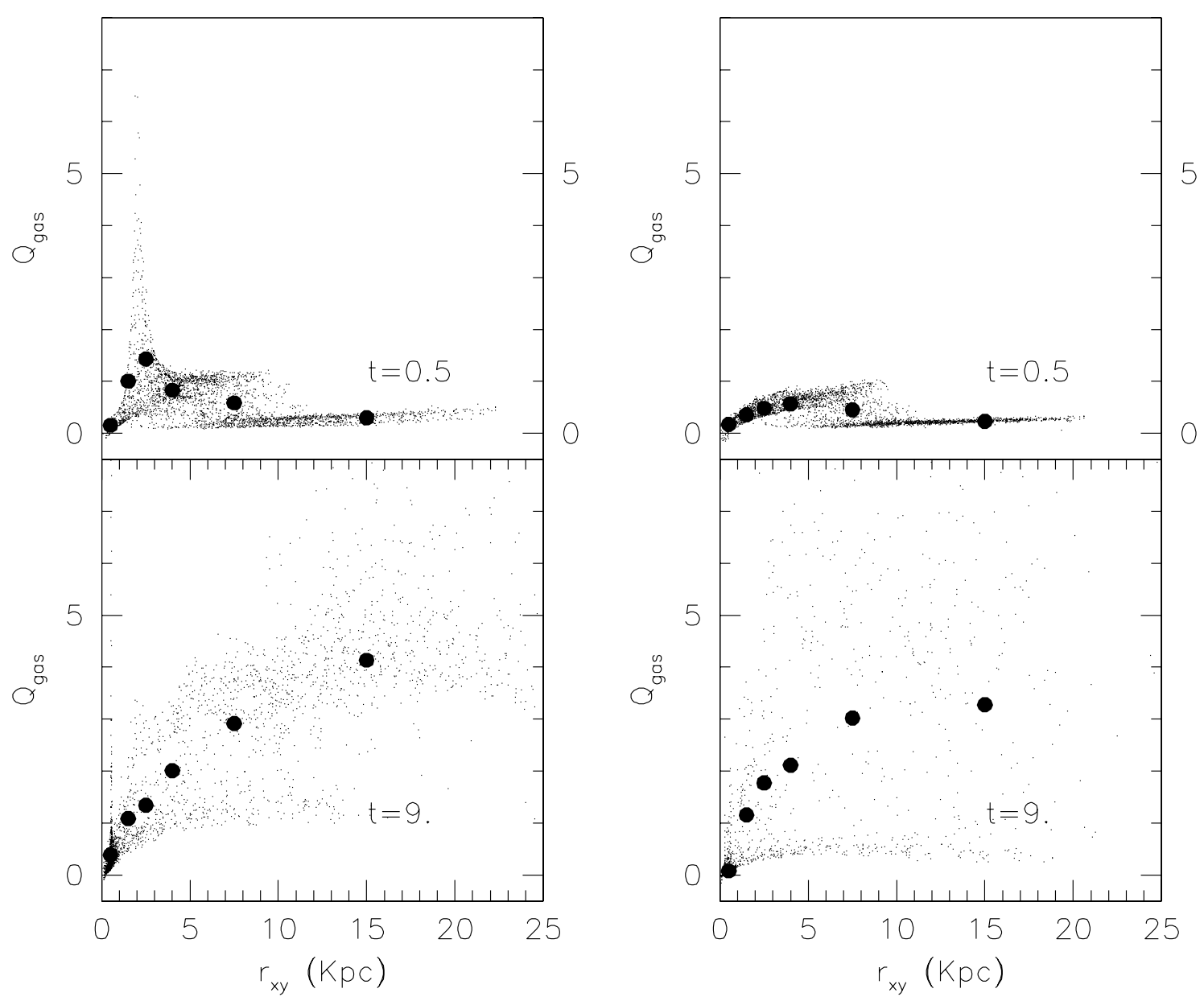

Fig. 4. $Q_{\mathrm{g}}$ distribution for simulations 2 (left panels) and 8 (right panels) in Table 1; filled points are $Q_{\mathrm{g}}$ average values computed inside the following radial steps: $0-1,1-2,2-3,3-5,5-10,10-20 \mathrm{kpc}$.

By comparing the global SEDs in Fig. 12 (continuous lines) we note their different shapes in the short wavelength spectral region, which depend on the residual SFR (see also Fig. 3), higher in simulation 1 than in simulation 2. This will provide different integrated colors. We find that after 1.7 Gyr the more concentrated haloes, which hold the stronger $B$ bars, show the shallowest color gradient (i.e. $\Delta(B-V)$ ) of new stars. This amounts to $0.15 \mathrm{mag}$ in simulation 1 and to $0.40 \mathrm{mag}$ in simulation 2 . Unrelaxed cases show larger color gradients slightly dependent on the total mass of the halo: $0.30 \mathrm{mag}$ and $0.40 \mathrm{mag}$ for simulation 7 and 8 respectively and $0.40 \mathrm{mag}$ 

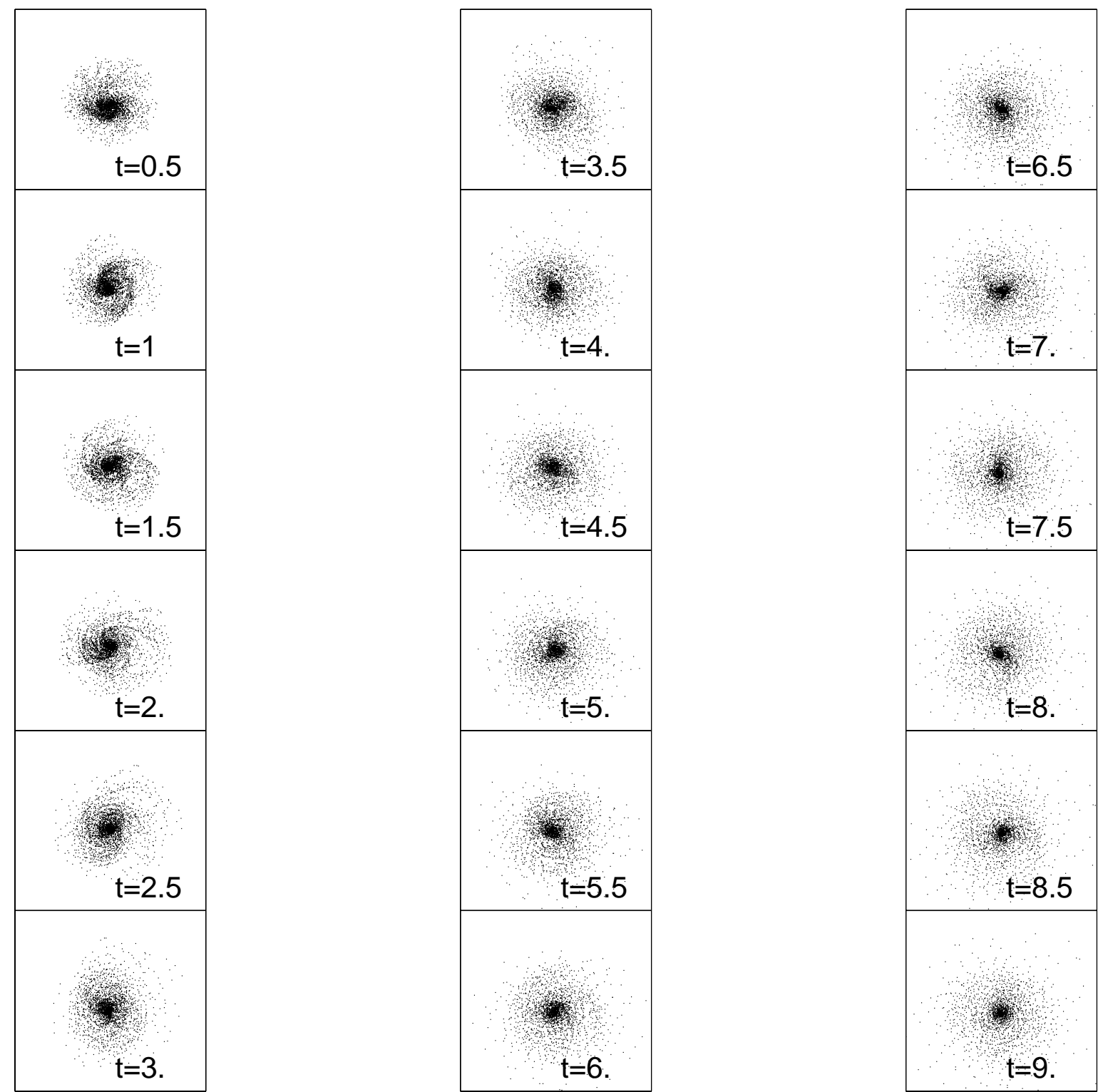

Fig. 5. Gas morphologies ( $x-y$ plane) for simulation 1 in Table 1 for a total spatial range of $40 \times 40 \mathrm{kpc}$.

and $0.51 \mathrm{mag}$ for simulation 10 and 11 . Moreover, slopes of metallicity gradients of new stars are considerably reduced in systems with stronger bars, in agreement with observations (Martin \& Roy 1994). Table 3 summarizes the results for the new star component in all the simulations performed.

\section{Discussion}

In this paper we have focused how the previous results (CM99) can be affected by the star formation process. We have discovered that a bar structure lasting at least 1.7 Gyr in the stellar component appears also in the more massive relaxed systems. Thus new stars have an important dynamical role in the global evolution, which cannot be neglected. Moreover, all the star components (the newly formed and the old ones) show a bar more clearly inside haloes having lower initial triaxiality and higher concentrations (Tables 2 and 3 ). The understanding of the behavior of angular momentum exchange between the different components of the system can explain such a point. Tables 4 and 5 (which relies on simulations without star formation implemented to account for the same cases here analyzed) tell us that the gas component exchanges more angular momentum if the star formation is switched on since a large portion of its initial angular momentum is transferred to the new star component (Table 4, Cols. 5 and 9). In the more massive relaxed systems the old stellar component exchanges less angular momentum here than in the case without star formation, since dynamical friction is reduced. This is a consequence of the lower mass density (Binney \& Tremaine 1987) following the star formation process, since dissipative particles can be closer than non-dissipative ones. The angular momentum loss from gas and stars becomes a gain for the DM. Therefore we find a minor dynamical coupling in the relaxed cases 

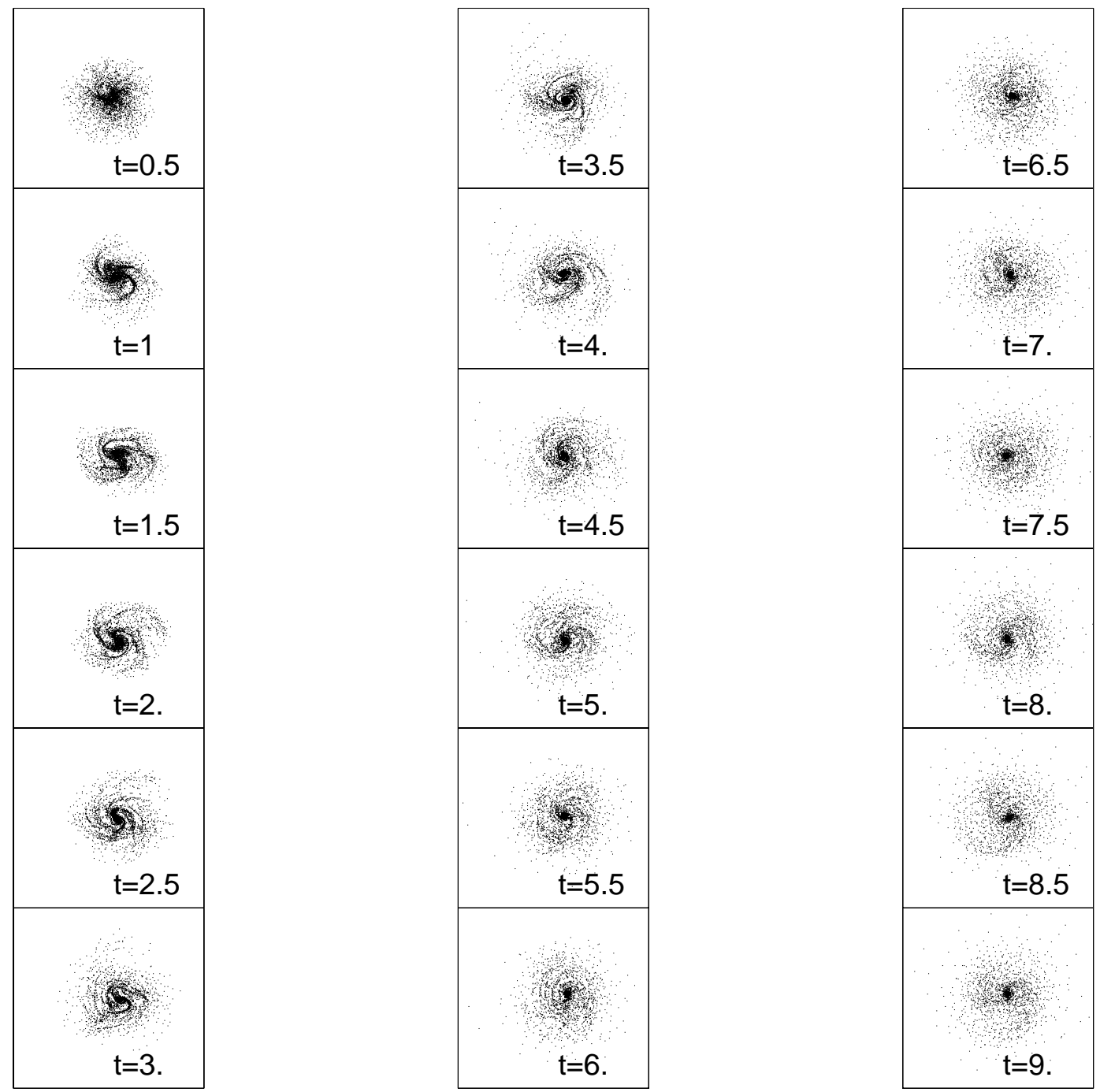

Fig. 6. Gas morphologies $(x-y$ plane) for simulation 2 in Table 1 for a total spatial range of $40 \times 40 \mathrm{kpc}$.

Table 4. Simulations with star formation switched on: percentages of both total and relative angular momentum exchange, $(\Delta L)_{\mathrm{i}} / L_{\mathrm{t}}$ and $(\Delta L)_{\mathrm{i}} / L_{\mathrm{i}}$ respectively, from the beginning to the final stage (i.e. $\left.t=10\right)$ of different components, (i), where $(\Delta L)_{\mathrm{i}}=L_{\mathrm{i}}(t=0)-L_{\mathrm{i}}(t=10)$.

\begin{tabular}{ccccccccc}
\hline $\mathrm{N}$ & $(\Delta L)_{\mathrm{i}} / L_{\mathrm{t}}: \mathrm{DM}$ & $\mathrm{Gas}$ & $\mathrm{Old}_{\mathrm{s}}$ & $\mathrm{New}_{\mathrm{s}}$ & $(\Delta L / L)_{\mathrm{i}}: \mathrm{DM}$ & $\mathrm{Gas}$ & $\mathrm{Old}_{\mathrm{s}}$ & $\mathrm{New}_{\mathrm{s}}$ \\
\hline 1 & +1.62 & -0.34 & -1.34 & +0.06 & +2.4 & -11.4 & -4.7 & +2.0 \\
2 & +0.59 & -0.35 & -0.67 & +0.43 & +0.8 & -16.0 & -3.2 & +20.0 \\
3 & +0.55 & -0.34 & -0.39 & +0.18 & +0.7 & -15.8 & -2.2 & +9.6 \\
4 & +3.48 & -1.77 & -2.59 & +0.88 & +16.6 & -23.5 & -3.6 & +12.0 \\
5 & +5.89 & -1.73 & -5.65 & +1.49 & +23.4 & -24.5 & -8.3 & +21.1 \\
6 & +2.20 & -0.75 & -2.20 & +0.75 & +8.0 & -11.3 & -3.4 & +11.3 \\
7 & +7.98 & -1.43 & -6.88 & +0.33 & +10.5 & -61.4 & -31.5 & +15.0 \\
8 & +6.50 & -1.40 & -5.40 & +0.30 & +8.2 & -70.0 & -28.1 & +14.0 \\
9 & +2.88 & -0.72 & -2.39 & +0.23 & +3.5 & -41.9 & -14.6 & +13.9 \\
10 & +5.70 & -1.80 & -5.60 & +1.70 & +25.0 & -25.2 & -8.0 & +24.0 \\
11 & +6.10 & -2.40 & -5.40 & +1.70 & +23.6 & -23.4 & -8.1 & +23.7 \\
12 & +3.90 & -1.75 & -3.55 & +1.40 & +13.3 & -26.2 & -5.7 & +20.0 \\
\hline
\end{tabular}

$L_{\mathrm{t}}$ is the total angular momentum of the system (constant).

Columns (5) and (9) show the difference between the initial angular momentum of the gas and the final one of new stars divided by $L_{\mathrm{t}}$ and $L_{\text {gas }}(t=10)$ respectively in percentage. 
P. Mazzei and A. Curir: Bar instabilities: The star formation onset
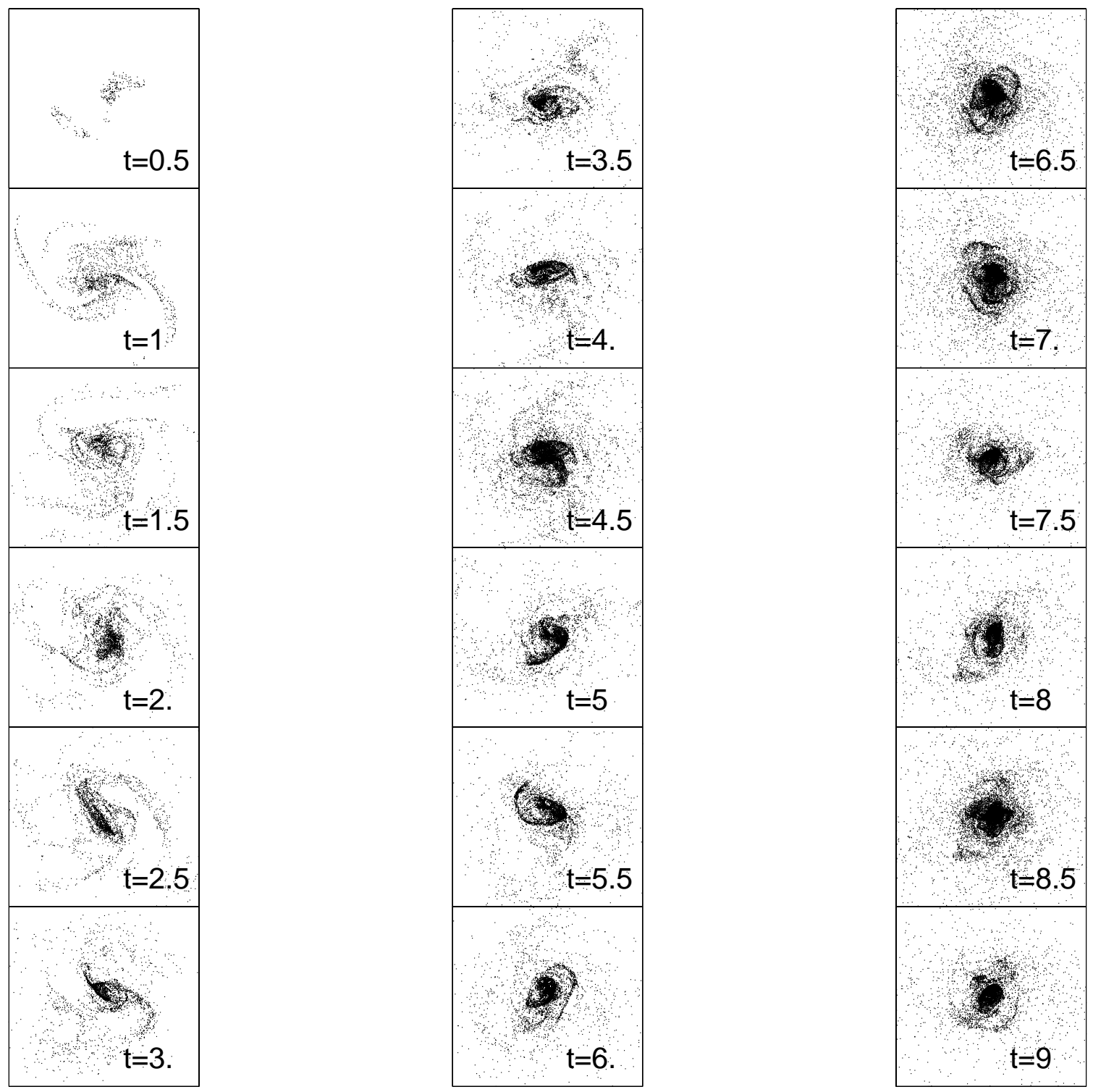

Fig. 7. Morphologies $(x-y$ plane) for new stars in simulation 2 (Table 1 ); the spatial range is $10 \times 10 \mathrm{kpc}$.

Table 5. Simulations with star formation switched off: percentages of both total and relative angular momentum exchange from the beginning to the final stage of different components.

\begin{tabular}{lrllcll}
\hline $\mathrm{N}$ & $(\Delta L)_{\mathrm{i}} / L_{\mathrm{t}}: \mathrm{DM}$ & $\mathrm{Gas}$ & $\mathrm{Old}_{s}$ & $(\Delta L / L)_{\mathrm{i}}: \mathrm{DM}$ & $\mathrm{Gas}$ & $\mathrm{Old}_{\mathrm{s}}$ \\
\hline 1 & +1.53 & -0.03 & -1.50 & +2.4 & -1.0 & -5.2 \\
2 & +1.20 & +0.05 & -1.25 & +1.6 & -2.3 & -6.0 \\
3 & +0.96 & -0.28 & -0.66 & +1.2 & -14.9 & -3.7 \\
4 & +4.01 & -0.70 & -3.31 & +19.1 & -9.3 & -4.8 \\
5 & +4.42 & -1.29 & -3.13 & +17.5 & -18.7 & -4.6 \\
6 & +1.19 & -0.84 & -0.35 & +4.3 & -12.3 & -0.5 \\
7 & +8.44 & -1.43 & -7.01 & +11.1 & -62.4 & -32.1 \\
8 & +6.23 & -1.43 & -4.80 & +8.0 & -71.5 & -25.0 \\
9 & +3.27 & -0.61 & -2.66 & +4.0 & -35.5 & -16.3 \\
10 & +6.15 & -0.75 & -5.40 & +26.9 & -10.0 & -7.8 \\
11 & +3.24 & -0.77 & -2.47 & +12.1 & -10.8 & -3.7 \\
12 & +6.53 & -1.00 & -5.53 & +22.2 & -14.5 & -8.9 \\
\hline
\end{tabular}

The meaning of the symbols is the same as in the previous table. 

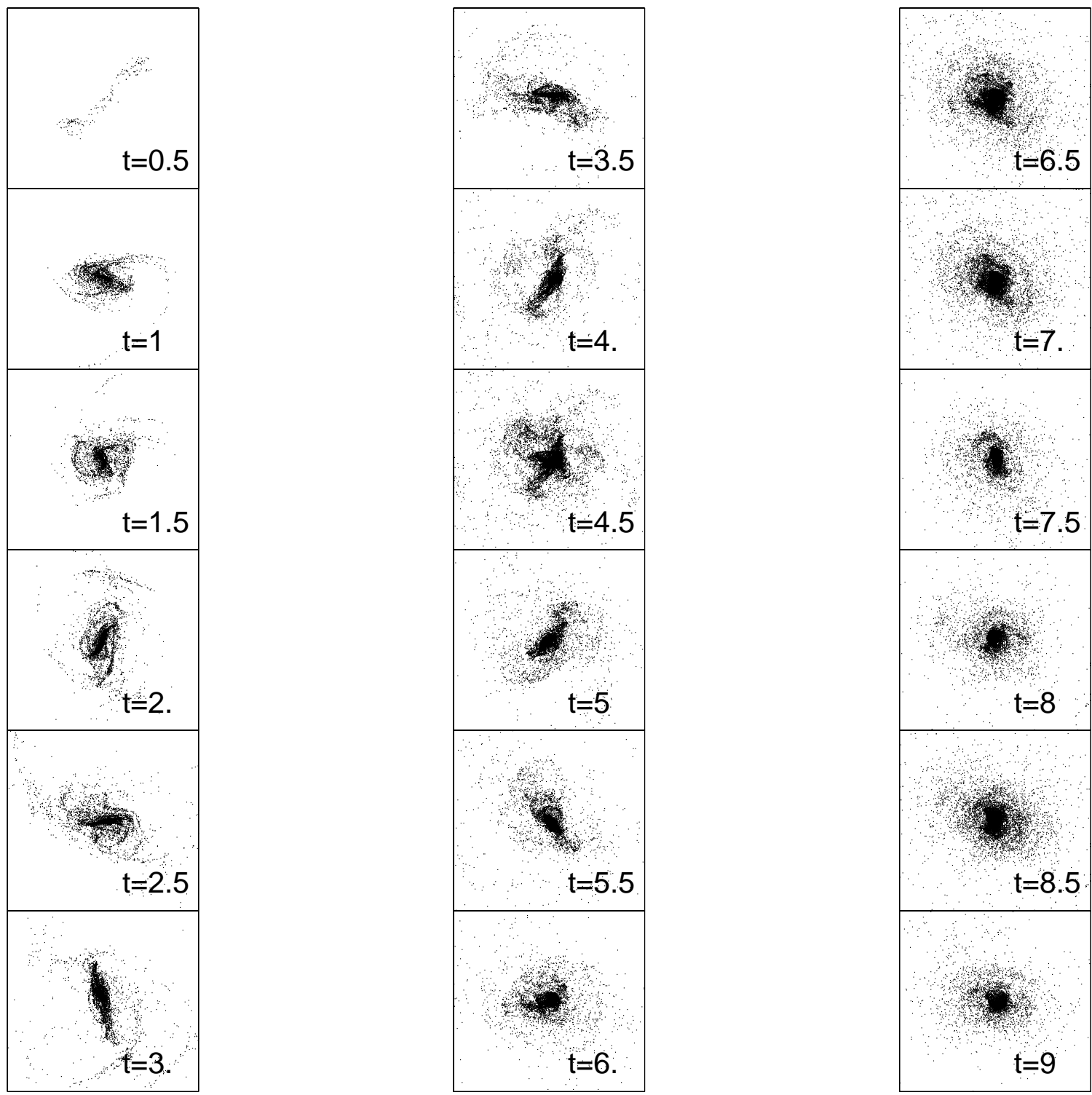

Fig. 8. Morphologies $(x-y$ plane) for new stars in simulation 8 (Table 1 ); the spatial range is $10 \times 10 \mathrm{kpc}$, as in the previous figure.

between baryonic and DM particles if the star formation is switched on. This affects the global stability of the disk which cannot re-arrange on the same dynamical time as the halo. Therefore disk instabilities can be maintained and are growing for longer time if the SFR is switched on also in the more stable conditions previously analyzed.

Friedli \& Benz (1993, 1995) and Martinet \& Friedli (1997) have studied the effect of star formation on the bar instability, though in a very different framework. Their models account for the baryonic component alone, the disk being stabilized by a stellar bulge of different mass ratios as compared to the disk or by a fixed halo. Friedli \& Benz (1995) find that star formation works towards the growth of bar instability by progressively adding dynamically cool masses in the stellar disc. Thus the star formation lengthens the life-time of the bar. Martinet \& Friedli (1997) also estimated that as much as $75 \%$ of the gas in the bar is not transformed in stars. Our more massive relaxed systems show, in the inner regions $(r<5 \mathrm{kpc})$, a residual gas fraction ranging from $80 \%$ to $51 \%$ as a consequence of their different DM concentration which affects the SFR.

An important result of this paper is indeed that the SFR, which depends on local conditions of the gas, is however governed by the global dynamics of the galaxies. The dynamical state of the DM halo affects the star formation process. We pointed out that the more massive relaxed haloes host disks with the lower SFR, whereas the opposite is true for the unrelaxed haloes having the higher masses (Table 3). This effect is enroled by the density of the halo which warms up the gaseous disk so that the conditions for the star formation become less favourable (Sect. 4). However the halo average density depends on its dynamical state but also on the initial geometry of the halo itself. In fact it has been shown (Sect. 3.1) that the halo concentration increases with the decrease of the initial triaxiality ratio $(\tau)$. The effect of the geometry on the strength of the 


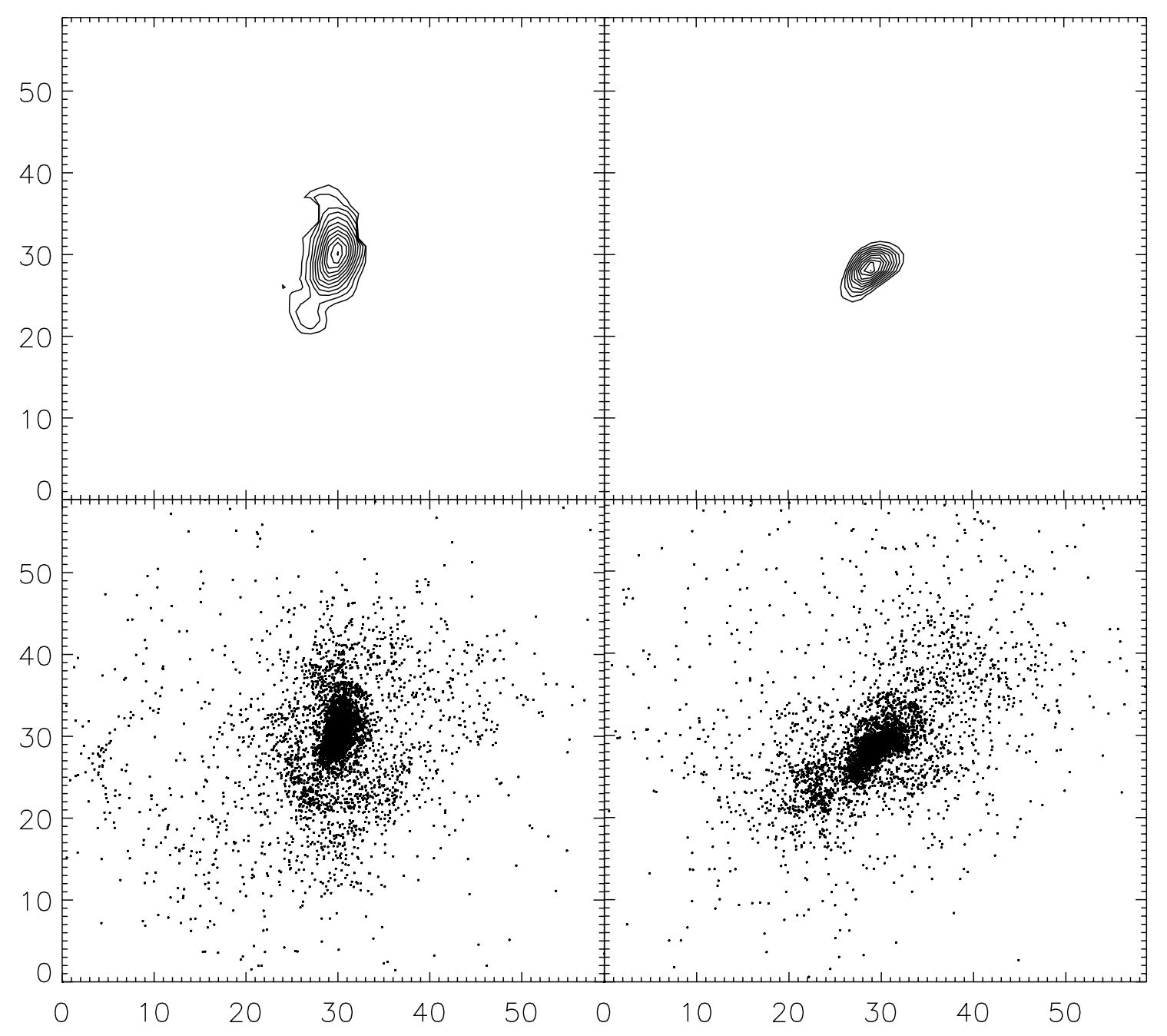

Fig. 9. Upper panels show $12 B$ isophotes of simulation 7 (Table 1) at $t=8$ and $t=9$ (left and right respectively); the magnitude contrast achieved, $5 \mathrm{mag}$, corresponds to a lower surface brightness contour 3 times higher than the minimum contour allowed by the maps; the spatial range $(x-y$ plane $)$ is $20 \times 20 \mathrm{kpc}$ and the resolution $\simeq 300 \mathrm{pc}$; lower panels show the corresponding morphologies. The number of new star particles is 10994 and 11652 respectively.

bar seems to play a further interesting role since stronger bars develop in the more concentrated haloes. We speculate that these arise at the final stage of such systems as a consequence of higher final SFR which occurs in their central regions (Sect. 4). Bars in bars, which we recover more easily in the less massive unrelaxed systems here analyzed, would reflect an instability growing on a spatial scale larger than in more massive relaxed systems, as a consequence of the lower initial concentration of less massive cases.

We performed a systematic analysis of the evolution of the triaxiality ratio, $\tau$, during the collapse and relaxation of the haloes presented in this paper. The $\tau$ parameter was calculated by plugging in the formula (1) the principal inertial axes of the system. We notice that isolated unrelaxed haloes settle in an asymptotic prolateness ranging between $\tau=0.7$ and $\tau=0.9$. However this occurs after $t=200$ in our numerical units. Thus it is not surprising that unrelaxed haloes here analyzed, which differ from isolated ones only for the embedded baryonic disk of $M=1$, do not show any defined trend since, as we said about the isolated haloes, a clear tendency to a defined value of $\tau$ arises only after $t=200$, which corresponds to many dynamical times (Table 1). In the case of the relaxed haloes, where the disk immersion was accomplished after halo evolution of 5 dynamical times (Sect. 3), we find that the initially spherical systems tend toward a triaxiality ratio defined by a $\tau \simeq 0.5,0.6$ at $t=10$, whereas if the halo has already a $\tau \geq 0.6$ its triaxiality keeps oscillating, by almost $0.17 \%$, around $\tau=0.6$. Thus the dynamical state of the halo also strongly affects the evolution of this ratio.

Our chemo-photometrical implementation of the code allows to derive colors and isophotes from UV to $K$ band of new stars in our set of simulated systems. We find that after 1.7 Gyr the more concentrated the halo, the stronger the $B$ bar of new stars, the shallowest their color and metallicity gradients, in agreement with observations (Martin \& Roy 1994). 


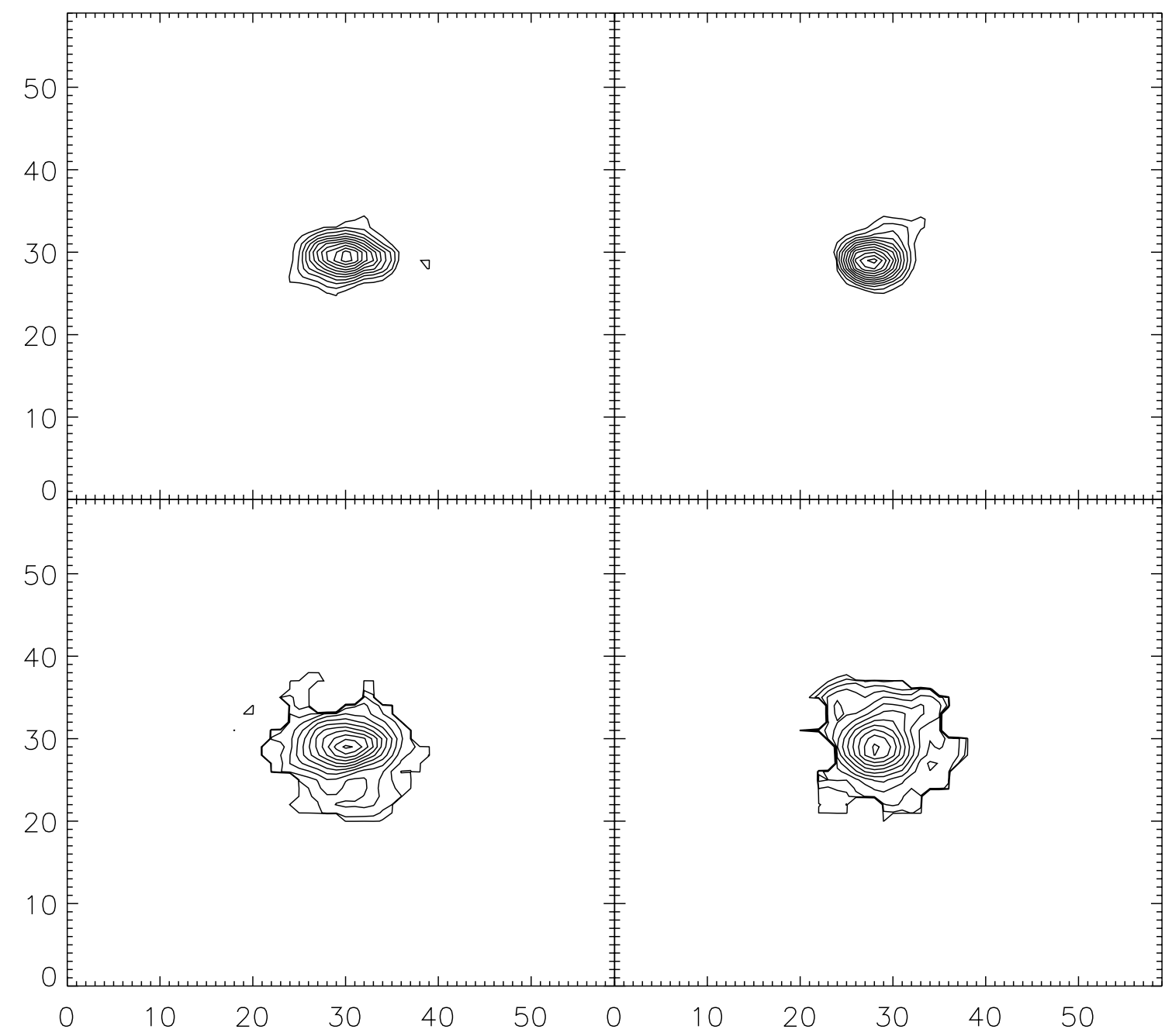

Fig. 10. 12 isophotes at $t=9$ of simulation 3 and 9 (Table 1), left and right column respectively, are shown; top panels show $B$ maps, bottom ones K maps; the magnitude contrast achieved, 5 mag, corresponds to a lower surface brightness contour at least 3 times higher than the minimum contour allowed by the maps; the $x-y$ spatial range and the resolution achieved are also the same as in the previous figure.

Interesting comparisons arise not only between the different appearance of the simulated galaxies in different bands but also between isodensity contours and isophotes of new stars. We point out that isophotes are contours of equal luminosity density instead of equal particle density. The comparison between Figs. 10 and 13 stresses how a link between results of a pure dynamical approach, i.e. isodensity contours, and $B$ or $K$ isophotes, as derived by our chemo-photometric predictions, is difficult; the comparison with observations is complicated further because bars are features strongly dependent on the spatial resolution and magnitude contrast achieved by instrumental performances and reduction techniques. We find that by increasing the spatial resolution we must increase the magnitude contrast by the same factor to achieve almost the same morphological details in our $B$ maps.

Dust effects can reflect further on the appearance of the bar, especially in the shortest wavelength spectral regions. In a forthcoming paper we will discuss this point in detail by comparing IR and FIR maps of our models with results of recent surveys (ISO).

\section{Conclusions}

In this paper we have analyzed the star formation onset in a stellar-gaseous disk embedded in live dark matter haloes of different mass, initial geometry and dynamical state.

We find important connections between dark and luminous matter. Our results show that:

i) the star formation rate (SFR) as well as the growth of bar instability in the baryonic disk depends both on the mass and on the dynamical state of the halo; its initial geometry further influences the final results. More massive relaxed systems achieve a lower SFR while the more massive unrelaxed haloes lead to a higher SFR;

ii) The formation of new stars reduces the dynamical coupling between the DM halo and baryonic disk so that star and gas components require more time to re-arrange 

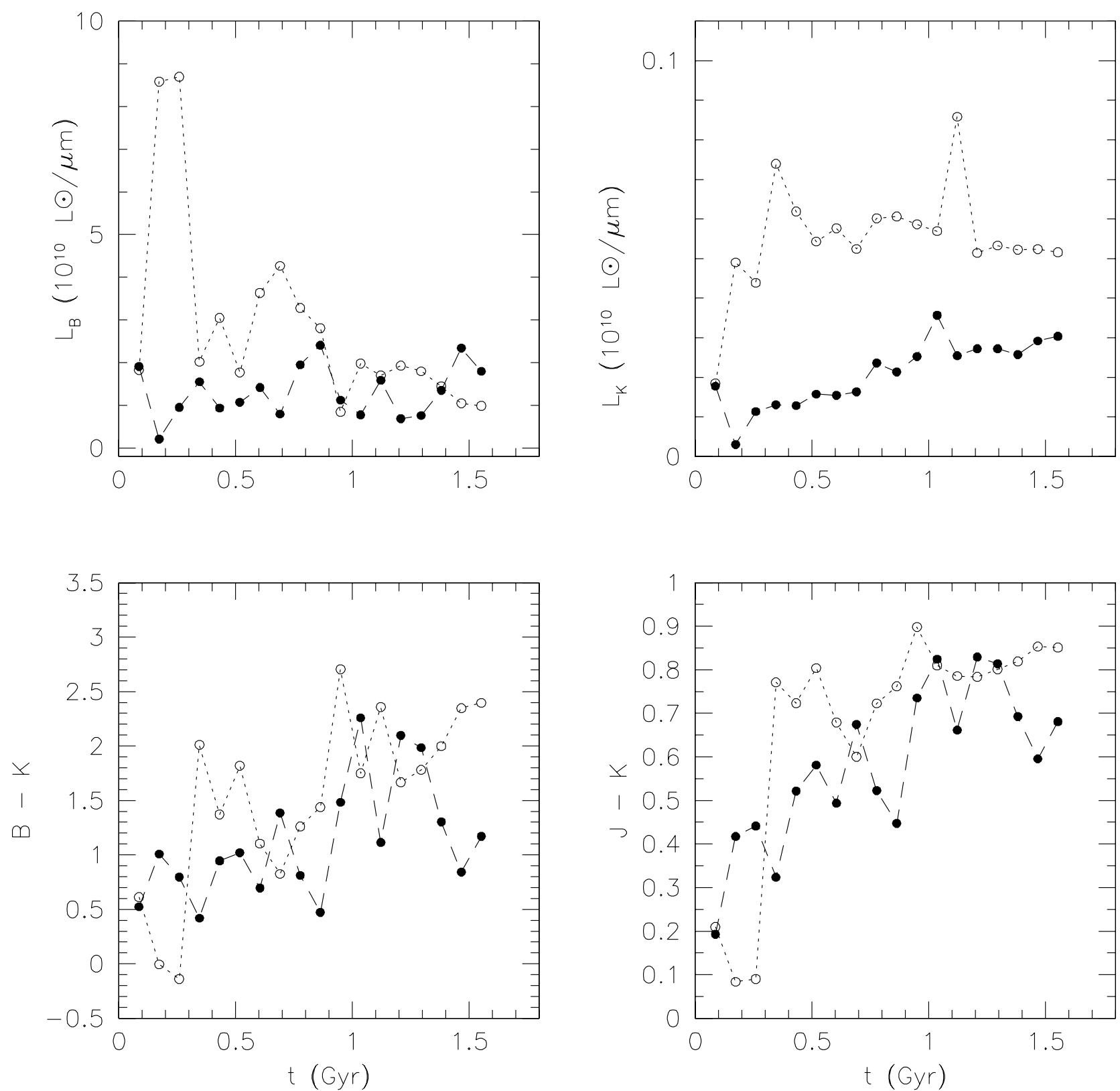

Fig. 11. Filled circles and dashed lines are for simulation 1 in Table 1, open circles and dotted lines for simulation 2; upper panels compare $B$ and $K$ luminosities, lower panels $B-R$ and $B-K$ colors at different times.

in a disk. Therefore a longer bar instability arises from star formation, lasting at least $1.7 \mathrm{Gyr}$, which develops in all the simulations here performed, and also in the more stable conditions previously analyzed (CM99);

iii) At the final time, stronger bars prefer more concentrated haloes, i.e. more massive systems with a lower initial triaxiality ratio; this is a consequence of their higher residual SFR due to the larger amount of residual gas;

iv) From our chemo-photometric implementation, we derive that these systems have short central bars ( $\leq 3-4 \mathrm{kpc})$ brighter in $B$ than other systems;

v) The more concentrated the halo, the shallower are metallicity and color gradients of new star formed, which also depend on the total mass of the halo itself: more massive unrelaxed haloes involve larger color gradients;

vi) By mapping the simulated systems in different bands, from UV up to near-IR wavelengths, we emphasize that the appearance of the bar is different if isophotes or isodensity contours are considered. Moreover, both the appearance and the strength of the bar are different in different bands for a given spatial resolution and magnitude contrast adopted. Dust effects can also affect the appearance of the bar, especially in the shortest wavelength spectral regions.

The results obtained in this paper claim that the astrophysical problem of bar instability is more than a purely dynamical problem. The understanding of the formation 

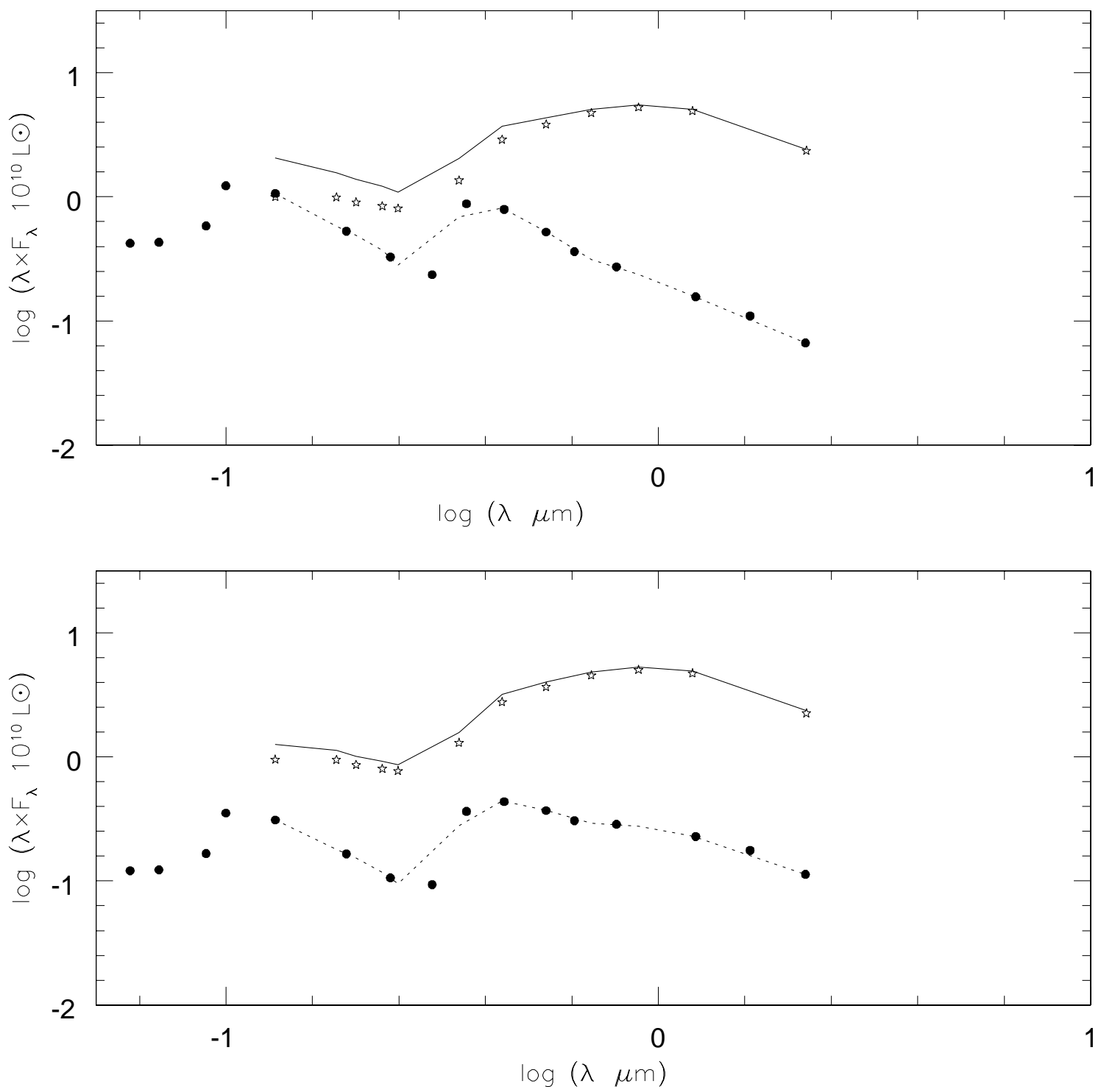

Fig. 12. Upper panel for simulation 1 in Table 1, lower panel for simulation 2; filled circles show the SED of the new stars after 1.6 Gyr whereas "stars" that of the old star component (see text), the continuous lines are the global (i.e. new+ old stars) SED.

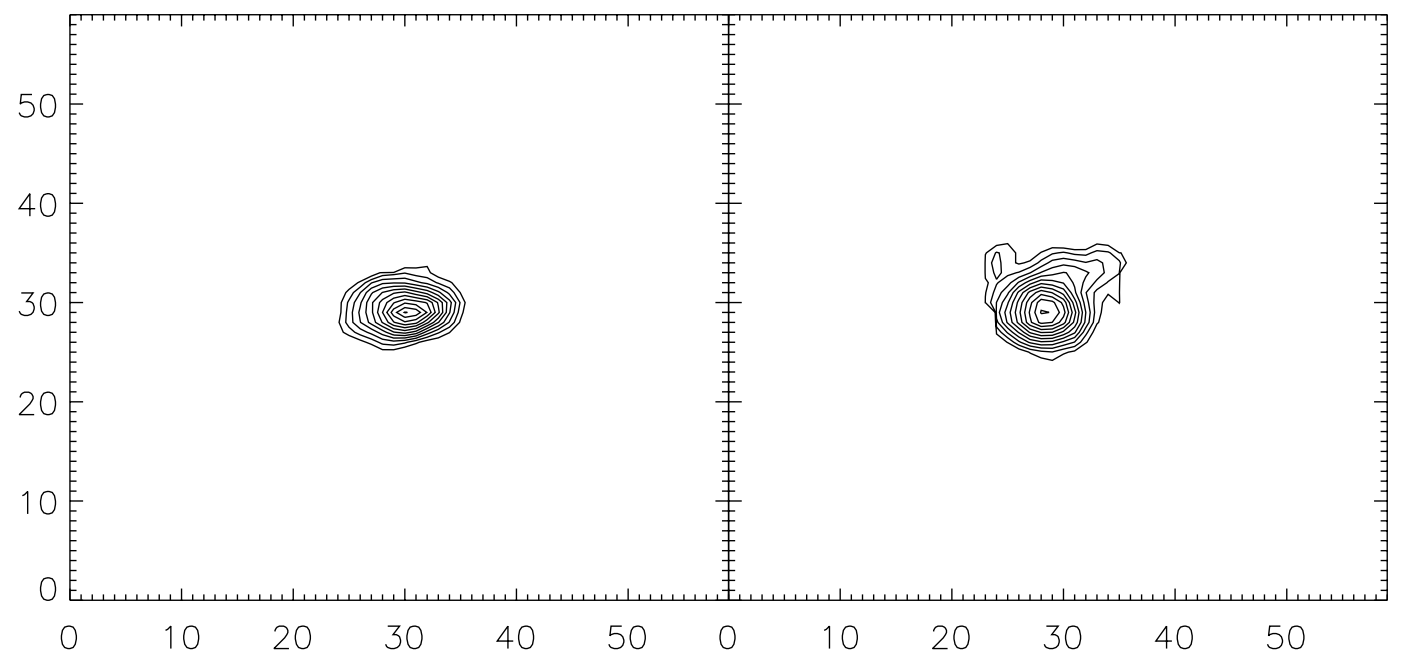

Fig. 13. 12 isodensity contours $(x-y$ projection) for the new stars of simulation 3 (left panel) and 9 (right panel) in Table 1 at $t=9$; the density contrast $(100)$, the spatial range $(20 \times 20 \mathrm{kpc})$ and the resolution $(\simeq 300 \mathrm{pc})$ are the same as in Fig. 10 . 
and evolution of bars inside host galaxies must move on different paths focusing both on the cosmological and dynamical framework, and on the effects of star formation, i.e. luminosity, chemical abundances and gradients, and on how these paths interplay.

\section{References}

Athanassoula, E., Bosma, A., \& Papaioannou, S. 1987, A\&A, 179,23

Barnes, J., \& Efstathiou, G. 1987, ApJ, 319, 575

Binney, J, \& Tremaine, S. 1987, Galactic Dynamics (Princeton Univ. Press, Princeton, New Jersy)

Christodoulou, D. M., Shlosman, I., \& Tohline, J. E. 1995, ApJ, 443, 551

Curir, A., \& Mazzei, P. 1999a, A\&A, 352, 103 (CM99)

Curir, A., \& Mazzei, P. 1999b, New Astron., 4, 1, Paper II

Debattista, V. P., \& Sellwood, J. A. 2000, ApJ, 543, 704

Efstathiou, G., Lake, G., \& Negroponte, J. 1982, MNRAS, 199, 1069

Friedli, D., \& Benz, W. 1993, A\&A, 268, 65
Friedli, D., \& Benz, W. 1995, A\&A, 312, 761

Friedli, D., Wozniak, H., Rieke, M., et al. 1996, A\&AS, 118, 461

Martinet, L., \& Friedli, D. 1997 A\&A, 323, 363

Mazzei, P., Xu, C., \& De Zotti, G. 1992, A\&A, 256, 45

Martin, P., \& Roy, J. R. 1994, ApJ, 424, 599

Martin, P. 1995, AJ, 109, 2428

Martin, P., \& Friedli, D. 1997, A\&A, 326, 449

Martin, P., \& Friedli, D. 1999, A\&A, 346, 769

Navarro, J. F., Frenk, C. S., \& White, S. D. M. 1995, MNRAS, 275,56

Ostriker, J. P., \& Peebles, P. J. E. 1973, ApJ, 186, 487

Sackett, P. 1999, Galaxy Dynamics, ed. D. Merritt, J. A. Sellwood, \& M. Valluri, ASP Conf. Ser., 182, 393

Sellwood, J. A. 1981, A\&A, 99, 362

Toomre, A. 1981, in Structure and evolution of normal galaxies, ed. S. M. Fall, \& D. Lynden Bell (Cambridge University Press, Cambridge), 111

Warren, M. S., Quinn, P. J., Salmon, J. K., \& Zurek, W. H. 1992, ApJ, 399, 405

Wozniak, H., Friedli, D., Martinet, L., Martin, P., \& Bratschi, P. 1995, A\&AS, 111, 115 